\title{
Anaesthesia for Ambulatory Paediatric Surgery: Common techniques and complications
}

\author{
Anesthésie pour ambulatoires de chirurgie pédiatrique: \\ Les techniques communes et les complications
}

\author{
C. Imarengiaye* ${ }^{* \dagger}$, D. Osifo ${ }^{\ddagger}$, S. Tudjegbe ${ }^{\dagger}$, I. Evbuomwan
}

\begin{abstract}
BACKGROUND: Ambulatory surgical care accounts for over $\mathbf{7 0 \%}$ of elective procedures in Northern America. Ambulatory paediatric surgical practice is not widespread in Nigeria. This report examined clinical indicators for quality care in paediatric ambulatory surgery using common outcomes after day case procedures as benchmark.

METHODS: This was a cross-sectional study of children who were presented for ambulatory surgical care in the University of Benin Teaching Hospital. A standardized questionnaire was employed to record the age, gender, indication for surgery, type of anaesthesia, timelines for the surgery and associated complications.

RESULTS: A total of 93 patients had surgical procedures on ambulatory basis. The mean age of the patients was 4.1 ? 4.0yr and duration of surgical procedure $31.3 \pm 12.1 \mathrm{~min}$. The male/ female ratio was $3: 1$, and herniotomy was the most frequent procedure on ambulatory paediatric surgical care $60(64.5 \%)$. The common anaesthetic techniques employed in the paediatric ambulatory setting were spontaneous respiration with face mask $40(43 \%)$, Inhalation technique with tracheal intubations $31(33.3 \%)$, general anaesthesia with relaxant technique five $(5.4 \%)$, local infiltration with or without sedation eight $(\mathbf{8 . 6 \%})$, GA plus caudal block eight $(8.6 \%)$, and subarachnoid block one $(1.1 \%)$. The indicators of quality care were unanticipated admission (5.4\%), repeat hospital visit $(4.3 \%)$, readmission $(2.2 \%)$ and delayed discharge $(21.5 \%)$.

CONCLUSION: The practices of paediatric surgery on ambulatory services are feasible in our setting. The observable complications are within acceptable limits. The timelines in the scheduling and discharge appear not to be optimal for an effective ambulatory service. WAJM 2009; 28(5): 304-307.
\end{abstract}

Keywords: Ambulatory care, Paediatric surgery, Anaesthesia, Outcome.

\section{RÉSUMÉ}

CONTEXTE: soins chirurgicaux ambulatoires comptes depuis plus de $70 \%$ des procédures électives en Amérique du Nord. Pratique de la chirurgie ambulatoire pédiatrique n'est pas très répandue au Nigéria. Ce rapport examine les indicateurs cliniques de soins de qualité en chirurgie ambulatoires pédiatriques en utilisant des résultats communs après que les procédures de jour comme point de repère.

MÉTHODES: Il s'agissait d'une étude transversale d'enfants qui ont été présentées pour des services de chirurgie ambulatoire à l'Université du Bénin à l'hôpital universitaire. Un questionnaire standardisé a été utilisée pour noter l'âge, le sexe, l'indication pour la chirurgie, le type d'anesthésie, les échéances pour la chirurgie et des complications associées. RÉSULTATS: Au total, 93 patients avaient des procédures chirurgicales en ambulatoire. L'âge moyen des patients était de 4,1 ? 4.0yr et la durée de procédure chirurgicale, 31,3 \pm 12,1 min. Le ratio hommes / femmes était de 3:1, et herniotomy a été la procédure la plus fréquente sur les soins chirurgicaux ambulatoires pédiatriques 60 (64,5\%). Les techniques d'anesthésie communs employés dans le contexte ambulatoire en pédiatrie ont été la respiration spontanée avec masque 40 (43\%), technique d'inhalation avec intubation trachéale 31 (33,3\%), l'anesthésie générale avec la technique relaxant cinq $(5,4 \%)$, infiltration locale avec ou sans sédation huit (8,6\%), GA bloc caudal, plus de huit $(8,6 \%)$ et du bloc sousarachnö̈dien d'un $(1,1 \%)$.

CONCLUSION: Les pratiques de la chirurgie pédiatrique, sur les services ambulatoires sont réalisables dans notre milieu. Les complications sont observables dans des limites acceptables. Le calendrier de la programmation et la décharge semble pas être optimale pour un service ambulatoire efficace. WAJM 2009; 28 (5): 304-307.

Mots-clés: soins ambulatoires, la chirurgie pédiatrique, d'anesthésie, issue.

Departments of Anaesthesiology ${ }^{\dagger}$ and Surgery ${ }^{\ddagger}$, University of Benin Teaching Hospital, Benin City, Nigeria.

Correspondence: Dr. C. O. Imarengiaye, Department of Anaesthesiology, University of Benin Teaching Hospital, PMB 1111, Benin City. +2348023250775 esemama@yahoo.co.uk

Abbreviations: ENT, Ear Nose and Throat. 


\section{INTRODUCTION}

Most centres worldwide are engaged in the management of paediatric surgical cases on ambulatory basis because of its perceived advantages. About two decades ago, Postuma et al ${ }^{1}$ reported about $60 \%$ of ambulatory surgical care in their practice. The subspecialty of ambulatory surgical practice has undergone a tremendous change in its scope and practice and this number must have increased in the developed centres. The advantages of ambulatory surgical care notwithstanding, the practice of ambulatory surgical care is not widespread in the West African sub region. Earlier reports on ambulatory surgical care in the sub region were limited to description of the various authors experience with their adult patients, ${ }^{2-5}$ and in particular the paediatric population. ${ }^{6-9}$

A major concern in ambulatory surgical care is the maintenance of quality care when compared to in-patient procedures. ${ }^{10,11}$ To our knowledge, the quality of care of ambulatory surgery especially in the paediatric population has not been reported in the sub region. It is important therefore, to determine the clinical indicators of quality care of a growing ambulatory paediatric surgical care. At present, there is no study to evaluate the effectiveness of ambulatory paediatric surgical care in this sub region. Hence this prospective study examined the known benchmarks so as to determine the efficiency or otherwise of ambulatory surgical practice in our environment.

\section{SUBJECTS, MATERIALS, AND METHODS}

This was a cross-sectional study of children who presented for ambulatory surgical care in the University of Benin Teaching Hospital. The Hospital is a tertiary teaching hospital with an established paediatric surgical service in the southern part of Nigeria. The paediatric surgical unit has three specialist paediatric surgeons. Ambulatory paediatric surgical care began as an organized practice five years ago.

Patients are seen in the paediatric surgical outpatient clinic to determine eligibility for ambulatory care. The patients are thereafter listed among inpatients on the scheduled operating surgical lists. All patients for circumcision were excluded from this study. On the morning of surgery, patients are received in the holding area of the paediatric surgical ward with their parents. The surgeon reviews the patients again to exclude any contraindication to surgery which could have developed since the last visit to the clinic, then the patients are reviewed by the anaesthesia team. All patients found fit are called to the operating room as scheduled. Anaesthesia is induced as deemed appropriate by the attending anaesthetist on the list. After surgery, the patients are returned to the holding area until declared fit for discharge based on unit protocol.

All paediatric patients scheduled for ambulatory surgery consecutively from January 2006 through June 2007 were studied prospectively. An elective procedure was defined as one scheduled on the daily published operating list of the paediatric surgical unit. A day case was defined as an outpatient procedure where a patient was instructed to attend on the day of surgery, and was to be discharged on that same day, and so indicated on the published operating list. A standardized questionnaire was employed to record the age and gender of the patients, indication for surgery, surgical procedure and category of patient. For each patient, time of commencement of surgery, time of completion of surgery, anaesthetic technique, time of discharge and preoperative complications were also recorded.

\section{RESULTS}

A total of 93 patients had surgical procedure on ambulatory basis. The mean age of the patients was $4.1 \pm 4.0$ years and duration of surgical procedure was $31.3 \pm 12.1$ minutes. There were more males than females presenting for paediatric ambulatory surgery with a male/female ratio of $3: 1.93 .5 \%(n=87)$ surgical procedures were concluded before 1600 hours but $21.5 \%(n=20)$ were discharged after 1600 hours. Herniotomy was the most frequent procedure on ambulatory paediatric surgical care with $60(64.5 \%)$ as seen in Table 1.
Table 1: Common Procedures and Anaesthetic Techniques for Ambulatory Paediatric Surgery

\begin{tabular}{lr}
\hline Features & Frequency $(\boldsymbol{\%})$ \\
\hline Surgical Procedures & \\
Herniotomy & $60(64.5)$ \\
Excision Biopsy & $17(18.2)$ \\
Orchidopexy & $7(7.5)$ \\
Re-circumcision & $4(4.3)$ \\
Syndactyly & $2(2.2)$ \\
Others & $3(3.2)$ \\
Anaesthetic Techniques & \\
GA with face mask & $40(43.0)$ \\
GA with tracheal intubation & $31(33.3)$ \\
GA with muscle relaxant & $5(5.4)$ \\
GA and caudal block & $8(8.6)$ \\
Local anaesthesia & $8(8.6)$ \\
Subarachnoid block & $1(1.1)$ \\
\hline
\end{tabular}

The common anaesthetic techniques employed in the paediatric ambulatory setting were spontaneous respiration with face mask $40(43 \%)$, inhalation technique with tracheal intubation 31(33.3\%), general anaesthesia with relaxant technique five $(5.4 \%)$, local infiltration with or without sedation eight $(8.6 \%)$, GA plus caudal block eight $(8.6 \%)$, and subarachnoid block one $(1.1 \%)$. Table 2 shows the common complications and outcome of the ambulatory pediatric surgical service. Pain, dizziness, delayed recovery from anaesthesia and sore throats were the anaesthesia related complications. Haematoma was the only surgical complication seen in this study (Table 2).

Table 2: Complications and Outcomes of the Ambulatory Pediatric Surgical Setting

\begin{tabular}{lr}
\hline Feature & Number (\%) \\
\hline Complications & \\
Haematoma & $1(1.1)$ \\
Pain & $5(5.4)$ \\
Delayed recovery & $2(2.2)$ \\
Dizziness & $2(2.2)$ \\
Sore throat & $2(2.2)$ \\
Outcomes & \\
Delayed discharge & $20(21.5)$ \\
Unanticipated admission & $5(5.4)$ \\
Repeat hospital visit & $4(4.3)$ \\
Readmission & $2(2.2)$ \\
\hline
\end{tabular}


Outcome of the ambulatory pediatric setting shows that unanticipated admission occurred in five $(5.4 \%)$ patients for delayed recovery, pain, dizziness and other unspecified cases. While, pain, haematoma, postoperative nausea and vomiting were the reasons for repeat hospital visit in four patients. Re-admission occurred in two patients due to pain and dizziness. Delayed discharged occurred in $20(21.5 \%)$ of these causes.

\section{DISCUSSION}

This prospective cross-sectional study evaluated 93 children who had surgery in the ambulatory setting to determine the efficiency of the ambulatory paediatric surgical service. Our results show unanticipated admission rate of $5 \%$, repeat hospital visit and delayed discharge in $21.5 \%$. Unanticipated hospital admission is a measure use to determine the quality of care in the ambulatory setting. The rate of unanticipated admission in our centre is high when compared to previous reports. The reasons for the unanticipated admission were related to late discharge times. One of the surgeons had a late morning list which ran into the late afternoon. This may be the reason for the delayed discharge timelines seen in this study. Otherwise, unanticipated admission rate for surgical or anaesthetic factors was comparable with published reports. ${ }^{12,} 13$

Repeat hospital visit and readmission rates are other benchmarks for an efficient ambulatory service. Pain, dizziness, postoperative nausea and vomiting were significant factors in the repeat visit and readmission to the hospital. Development of clinical management strategies to deal with these problems may result in better outcome. In addition, these factors as seen in this study can be improved upon by the development of appropriate protocol for scheduling of cases. Early completion of surgery and appropriate discharge times would minimize these adverse effects.

The range of procedures performed were mainly from the paediatric surgical unit, and hence the restriction to general surgical procedures as seen in this study. However, this pattern is similar to previous reports on paediatric day case in Nigeria. ${ }^{7,8}$ Elsewhere, the scope of procedures is not limited to general surgical procedures as other subspecialties are involved. Nevertheless, general surgery still account for a large proportion of caseloads for paediatric ambulatory service in the advanced centres. ${ }^{14,15}$ Recently, Asiri et al ${ }^{16}$ demonstrated that paediatric ENT procedures could be done safely as day case procedures. Thus, our paediatric ambulatory service can be expanded in the future to accommodate more procedures beyond general surgery.

Various anaesthetic options are available for the care of the ambulatory paediatric patient. General anaesthesia was the major anaesthetic technique for patients in this study. A previous study had showed more anaesthesia related complications and longer recovery times after general anaesthesia than regional technique. ${ }^{12}$ Indeed, over three-quarters of the patient population had surgery amenable to regional techniques such as spinal or caudal epidural. Kokki and colleagues ${ }^{17}$ demonstrated the safety and effectiveness of spinal anaesthesia in 195 children aged six months to 10years undergoing herniotomy. It may have been expedient to use more of regional techniques including caudal block as against its occasional use in the study. Caudal technique augments intraoperative anaesthesia and provides good analgesia in the postoperative period.

Pain is a common complication after ambulatory paediatric surgical procedures $^{8,15,17-19}$. The incidence and severity of postoperative pain in the paediatric population is related to the surgical procedure. More children who had orchidopexy, reported pain than those who had herniotomy, prompting a suggestion of continuous use of analgesics up to two-three days postoperatively. ${ }^{20}$ This suggestion notwithstanding, the place of caudal block in pain control in children is clear. Caudal analgesia is the most useful and popular paediatric regional block in current use. ${ }^{21}$ Single shot caudal blocks are well suited for surgical procedures below the umbilicus, ${ }^{22}$ which might have been useful in a good proportion of the patients studied.
In conclusion, we studied prospectively 93 children who had surgery in an ambulatory setting. The rate of delayed discharge, unanticipated admission, repeat hospital visit and readmission were high. Improved scheduling of patients for ambulatory surgery may minimize these problems.

\section{REFERENCES}

1. Postuma R, Ferguson CC, Stanwick RS Horne JM. Paediatric daycare surgery: a 30year hospital experience. J Ped Surg 1987; 22: 304-7.

2. Awojobi DA, Sagua AC, Ladipo JK. Outpatient management of external hernia. A district hospital experience. West Afr J Med 1987; 6: 201-4.

3. Jiburum BC, Akpuaka FC. Scope and problems in day case surgery in a plastic surgical unit. West Afr J Med 1996; 15: 237-8.

4. Imarengiaye $\mathrm{CO}$, Ande $\mathrm{AB}$. Experience with ambulatory anesthesia in gynaecological patients. Niger Postgrad Med J 2000; 7: 116-9.

5. Nkyekyer K. Day case laparoscopy in a Ghanian Teaching Hospital. Trop Doct 1996; 26: 147-50.

6. Adeyemi SD, daRocha-Afodu JT, Olayiwola B. Outpatient herniotomy with ketamine. A prospective study of 50 herniotomized children and review of 219 herniotomies with ketamine. West Afr J Med 1985; 4: 155-61.

7. Yawe T, Dogo D, Abubakar Y. Day case surgery: Experience with inguinal and abdominal wall hernias in children. Nig Med Pract 1997; 33: 31-2.

8. Adejuyigbe O, Abubakar AM, Sawande $\mathrm{O}$, Olasinde OO. Day case surgery in children in Ile-Ife, Nigeria - an audit. Nig J Surg 1998; 5: 60-3.

9. Bode CO, Adeyemi SD. Reasons for day surgery cancellation in paediatric surgical practice at the Lagos University Teaching Hospital. Nig J Surg 1996; 3: 41-4.

10. Martel E, Bernard D, Tasse D, Wassef R. Ambulatory anal surgery: feasibility study. Ann Chir 1996; 50: 589-92.

11. Letts M, Davidson D, Splinter W, Conway P. Analysis of the efficacy of paediatric day surgery. Can J Surg 2001; 44: 193-8.

12. Osborne GA, Rudkin GE. Outcome of day-care surgery in a major teaching hospital. Anaesth Intensive Care 1993; 21: 822-7.

13. Awad IT, Moore M, Rushe C, Elburki A, O'Brien K, Warde D. Unplanned hospital admission in children 
undergoing daycase surgery. Eur $J$ Anaesthesiol 2004; 21: 379-84.

14. Murat I, Constant I, Maud'huy H. Perioperative anaesthetic morbidity in children: a database of 24165 anaesthetics over a 30-month period. Paediatr Anaesth 2004; 14: 158-66.

15. Hariharan S, Chen D, Merrit-Charles L, Rattan R, Muthiah K. Performance of a paediatric ambulatory anaesthesia program - a developing country experience. Paediatr Anaesth 2006; 16: 388-93.

16. Asiri AM, Abu-Bakr YA, Al-Enazi F. Paediatric ENT day surgery. Is it safe practice? J Ambulatory Surg 2006; 12:
147-149.

17. Kokki H, Heikkinen M, Ahonen R. Recovery after paediatric daycase herniotomy performed under spinal anaesthesia. Paediatr Anaesth 2000; 10: 413-7.

18. Kotniemi LH, Ryhanen PT, Valanne J, Jokela R, Mustonen A, Poukkula E. Postoperative symptoms at home following daycase surgery in Children: a multicentre survey of 551 children. Anaesthesia 1997; 52: 963-9.

19. Faponle AF, Usang UE. Postoperative symptoms at home in children following daycase surgery. Middle East Journal of Anaesthesiology 2007; 19:
185-96.

20. Ho D, Keneally JP. Analgesia following paediatric day-surgical orchidopexy and herniotomy. Paediatr Anaesth 2000, 10: 627-31.

21. Verghese ST, Hannallah RS. Postoperative pain management in children. Anesthesiol Clinic N. Am 2005; 23: 163 184.

22. Hannallah RS, Broadman LM, Belman $\mathrm{AB}$, Abramowitz MD, Epstein BS. Comparison of caudal with ilioinguinal/ iliohypogastric nerve blocks for control of post-orchidopexy pain in paediatric ambulatory surgery. Anesthesiology 1987; 66: 832-4. 